\title{
Detection of emission from Cygnus Cocoon above 100TeV with LHAASO
}

\section{Cong Li, ${ }^{a b, *}$ Songzhan Chen, ${ }^{a, b}$ Sha Wu, ${ }^{a, b}$ Lingyu Wang, ${ }^{a, b}$ Ruizhi Yang ${ }^{c}$ and Ruoyu $\mathrm{Liu}^{d}$ on behalf of the LHAASO Collaboration}

(a complete list of authors can be found at the end of the proceedings)

${ }^{a}$ Key Laboratory of Particle Astrophyics \& Experimental Physics Division \& Computing Center, Institute of High Energy Physics, Chinese Academy of Sciences, 100049 Beijing, China

${ }^{b}$ TIANFU Cosmic Ray Research Center, Chengdu, Sichuan, China

${ }^{c}$ University of Science and Technology of China, 230026 Hefei, Anhui, China

${ }^{d}$ School of Astronomy and Space Science, Nanjing University, 210023 Nanjing, Jiangsu, China

E-mail: licong@ihep.ac.cn,

The origin of PeV cosmic rays in the Galaxy is a long-standing puzzle. Superbubble or massive star cluster (MSC) has been suggested as powerful cosmic-ray accelerators. Cygnus Cocoon, an extended gamma-ray source, is likely associated with the MSC Cygnus OB2. Previous observations have shown that its spectrum extends up to $100 \mathrm{TeV}$ with a break around $10 \mathrm{TeV}$. The spatial coincidence between the gamma-ray emission and molecular clouds in the region implies the hadronic origin of the gamma-ray emission, supporting Cygnus cocoon as a cosmic-ray proton accelerator. However, lack of a precise measurement of the spectrum beyond $100 \mathrm{TeV}$ as well as relevant morphology prevent people from concluding that it is the source of $\mathrm{PeV}$ cosmic rays. We here report the observation of LHAASO-KM2A on this region. The maximum significance above $25 \mathrm{TeV}$ is about $21.8 \sigma$. A photo with energy up to $1.4 \mathrm{PeV}$ is detected from this region, which indicates the spectrum can extend up to $1 \mathrm{PeV}$. Such a result may be considered as the evidence for cosmic ray accelerated beyond $\mathrm{PeV}$ in Cygnus Cocoon.

$37^{\text {th }}$ International Cosmic Ray Conference (ICRC 2021)

July 12th - 23rd, 2021

Online - Berlin, Germany

\footnotetext{
*Presenter
} 


\section{Introduction}

It is widely believed that there are sources in our galaxy can at least accelerate particles to $\mathrm{PeV}$ ("PeVatrons"). However, there is a lack of direct evidence for existence of such sources. SNR was proposed as one of the most probable cosmic ray sources. There are two arguments supporting this hypothesis. One is that supernovae explosion has enough energy to contain the cosmic ray flux in galaxy. The other is the success of DSA (diffuse shock acceleration) mechanism. The first direct evidence for proton acceleration in SNR was provided by Fermi-LAT[1]. However, weather SNR can serve as PeVatron is still in doubt. In normal condition, it is hard for SNR to accelerate particles to PeV. From observation aspect, the spectrum of more than 10 young SNRs appear to be steep below $10 \mathrm{TeV}$ [2]. Superbubbles with most of the power from supernova in our Galaxy released inside are ideal candidates for galactic cosmic ray accelerators[3][4]. The energy spectrum and radial distribution of derived comic ray flux provide evidences for particles accelerated near to $\mathrm{PeV}$ in massive star cluster[2].

Cygnus region is the most active star forming region in the north sky. Cygnus Cocoon is an extended source located in Cygnus-X region. It was firstly reported by Fermi-LAT at GeV[5]. At $\mathrm{TeV}$ energies, it was identified by ARGO experiment at first[6]. HAWC extended the energy spectrum to $100 \mathrm{TeV}$ [7]. The spectrum of Cygnus Cocoon can be described by a power-law below $10 \mathrm{TeV}$, and it behaves a soft feature around 10TeV. Due to the large angular size of Cocoon, it was not detected by cherenkov telescope. Besides Cygnus Cocoon, there are multiple sources also located in Cygnus Cocoon region, including a middle-aged SNR $(\gamma$-Cygni) and a PWN(TeV J2032+4130). $\gamma$-Cygni is in the middle of the Sedov phase, and it shows a bright circular radio shell, which was firstly detected by VERITAS in VHE range[8]. TeV J2032+4130 was first detected by the HEGRA observatory and had been shown to favor a PWN model associated with PSR J2032+4127 by VERITAS [9]. The excellent sensitivity of LHAASO can give detail morphological and spectral measurement of Cocoon from $\mathrm{TeV}$ to $\mathrm{PeV}$, which is crucial for identification of PeVatron.

\section{LHAASO-KM2A experiment}

The Large High Altitude Air Shower Observatory(LHAASO) is a hybrid extensive air shower (EAS) array with an area of about $1 \mathrm{~km}^{2}$ at an altitude of $4410 \mathrm{~m}$ a.s.l. in Sichuan Province, China. The one square array(KM2A) consists of 5195 electromagnetic particle detectors (EDs) and 1188 muon detectors (MDs). This array is mainly designed to detect gamma rays and cosmic rays with energy above tens of $\mathrm{TeV}$. The EDs are used to reconstruct the main parameters of shower, such as direction, energy and so on. The MDs are used to discriminate electromagnetic showers from hadronic showers. The ED is a scintillation detector covered by a 5-mm-thick lead plate to absorb low-energy charged particles and to convert high energy $\gamma$-rays into electron-positron pairs. The MD is a water Cherenkov detector with ultra-pure water as detection medium. The detector is covered by overburden soil with thickness of $2.5 \mathrm{~m}$, which absorbs most of the secondary electron/positrons and $\gamma$-rays. More details about the detectors are presented in He [10]. About half of the detectors have been installed and started operation since the end of 2019.

The array is triggered once more than 20 EDs fired within a time window of $400 \mathrm{~ns}$. Then all hits within $5 \mu$ s before and after the trigger time will be recorded and for off-line analysis. With 
the calibration of each detector, the ADC counts of EDs and MDs are converted into number of particles. The details about ED calibration can be found elsewhere [11]. The calibration for MDs is similar to that of EDs, which is based on the reconstruction of shower front. The status of each detector is monitored in real time, and only detectors under normal conditions are used for reconstruction.

Both measured and simulated events are processed through the same reconstruction pipeline. The direction of showers is reconstructed by fitting the relative arriving time of the ED hits. The angular resolution (denoted as $\phi_{68}$, containing $68 \%$ of the events) is $0.24^{\circ}$ at $100 \mathrm{TeV}$ for gamma-ray showers with zenith angles less than $35^{\circ}$. The parameter $\rho_{50}$, defined as the particle density at 50 $\mathrm{m}$ from the shower axis obtained by fitting the NKG function to the shower hits, is used as energy estimator[12]. The energy resolution is about $13 \%$ at $100 \mathrm{TeV}$ for showers with zenith angle less than $20^{\circ}$. The ratio between muons and electrons is used to discriminate electromagnetic showers from hadronic showers. The rejection power is about $4 \times 10^{3}$ at energies above $100 \mathrm{TeV}$, and will be better at higher energies. The data reconstruction, calibration and selection are described detailed in Aharonian et al. [12].

\section{Data analysis and results}

Data collected from December 2019 to November 2020 by the half of KM2A was used in this analysis. The total effective observation time is 308.33 days after the data quality check. The same event selection is used as described in the performance paper [12]. One decade of energy is divided into 5 bins with a bin width of $\log _{10} E=0.2$ taking into account the energy resolution and statistics. The "direct integration method" [14], which uses events with the same direction in horizontal coordinates but at different arrival time, is adopted to estimate the number of cosmic ray background events.

The test statistic used to evaluate the significance of the test is TS $=2 \log (\lambda)$, where $\lambda=$ $\mathcal{L}_{s+b} / \mathcal{L}_{b} . \mathcal{L}_{s+b}$ is the maximum likelihood value for source plus background hypothesis, while $\mathcal{L}_{b}$ is the background-only hypothesis. According to Wilks' Theorem [13], TS follows chi-square distribution with the number of degrees of freedom equal to the difference of number of free parameters between the hypotheses. A two-dimension gauss model with sigma fixed at $0.3^{\circ}$ is used here. The significance map around cygnus cocoon region above $25 \mathrm{TeV}$ is shown in Fig.1. The maximum significance is more than $20 \sigma$. The center of the emission is close to both the PWN and $\mathrm{OB} 2$ cluster. Due to the complex composition of this region, a multi-source fitting procedure was adopted to fit the emission in this region simultaneously. Considering the PSF of KM2A, only events with energy above $25 \mathrm{TeV}$ is used in fitting. There are 4 energy bins used in this analysis. The position,extension and total number of events in each energy bin are free parameters, so there are 7 free parameters in total. The point spread function for each energy bin is considered. Benefiting from the excellent sensitivity of KM2A above tens of $\mathrm{TeV}$, we successfully distinguish the contribution from different sources in this complex region by fitting the emission in this region simultaneously. The emission is well described by three sources:A slightly extended source with Gaussian width of $0.24^{\circ} \pm 0.03^{\circ}$ at the center of the region $\left(\mathrm{RA}=307.98^{\circ} \pm 0.03^{\circ}, \mathrm{Dec}=41.42^{\circ} \pm 0.03^{\circ}\right)$, which maybe associated with the PWN TeV J2032+4130. A very extended source with Gaussian width of $2.8^{\circ} \pm 0.3^{\circ}$ and best fitted position at $\mathrm{RA}=308.2^{\circ} \pm 0.4^{\circ}$ and $\mathrm{DEC}=41.3^{\circ} \pm 0.3^{\circ}$, which is the 


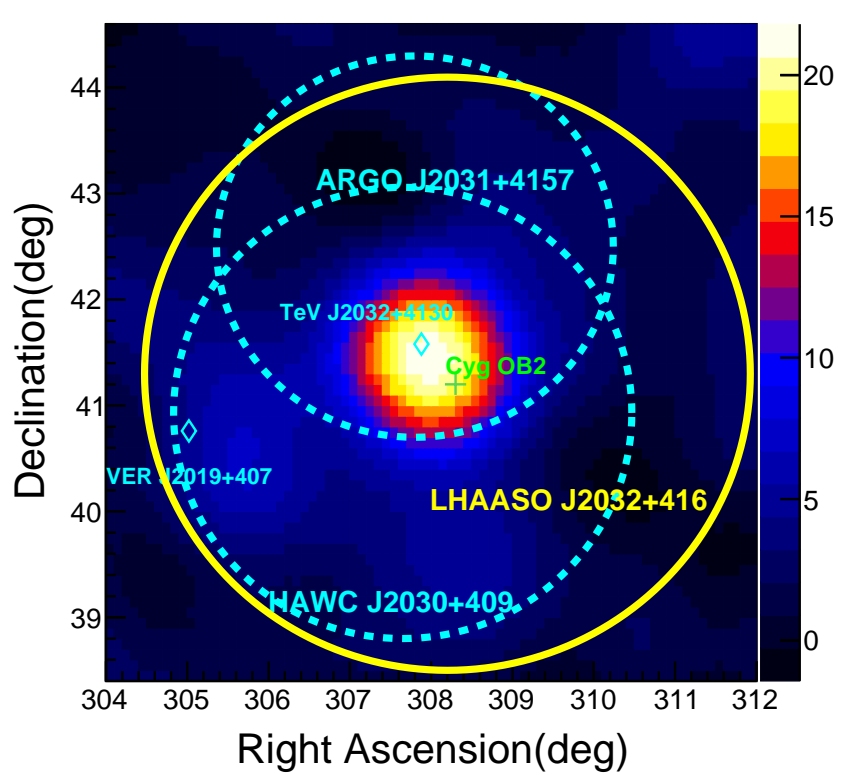

Figure 1: The significance map in Cygnus Cocoon region above $25 \mathrm{TeV}$. The blue diamonds marks $\mathrm{TeV}$ sources TeV J2032+4130 and VER J2019+407. The two blue dashed circle marks two very extended sources ARGO J2031+4157 and HAWC J2030+409. The yellow circle marks the source LHAASO J3032+416.

counterpart of $\mathrm{GeV}$ Cygnus Cocoon(shown in Fig.1). The third source is at $\mathrm{RA}=305.5^{\circ} \pm 0.2^{\circ}$ and $\mathrm{DEC}=40.4^{\circ} \pm 0.1^{\circ}$, which maybe associated with Gamma Cygni. The 3D likelihood framework is still under developing, thus the details about the morphology and spectrum for individual source is under studying now.

A photo with energy up to $1.4 \mathrm{PeV}$ was detected in this region, as reported in Cao et al. [18]. Important information can be obtained even from this single gamma ray event with the excellent rejection power and energy resolution of LHAASO. For this highest-energy photon, the measured ratio $\mathrm{N}_{\mu} / \mathrm{Ne}=1 / 941$. This low ratio rejects almost all $\mathrm{CR}$ background, the chance probability is estimated to be $0.028 \%$. The spillover effect in a wide energy range from $10 \mathrm{TeV}$ to few petaelectronvolts was further examined. The number of events in different reconstructed energy bins assuming a Crab Nebula SED with different cut energy are shown in Fig.3 . The spillover effect is determined by events in the bins above $E_{c u t}$. The spillover mainly happens in the adjacent bin, and the fraction of events in the next-adjacent bin is very small. The effect is also reduced with energy. The fraction runs lower than $1 \%$ for $E_{c u t}>400 \mathrm{TeV}$ even in the adjacent bin. So, it is clear that the energy spectrum can extend beyond $\mathrm{PeV}$ at least in the center of the emission, which can be understood as the evidence for particle accelerated beyond PeV.

\section{Conclusion}

Cygnus Cocoon is a very extended source located near the Cygnus OB2 cluster. The emission is likely to be produced by cosmic rays accelerated in Cygnus OB2 cluster. With the operation of half of KM2A, the maximum significance above $25 \mathrm{TeV}$ is more than $20 \sigma$. A PeV photo is successfully detected near OB2 cluster, which maybe the first evidence for cosmic ray accelerated 


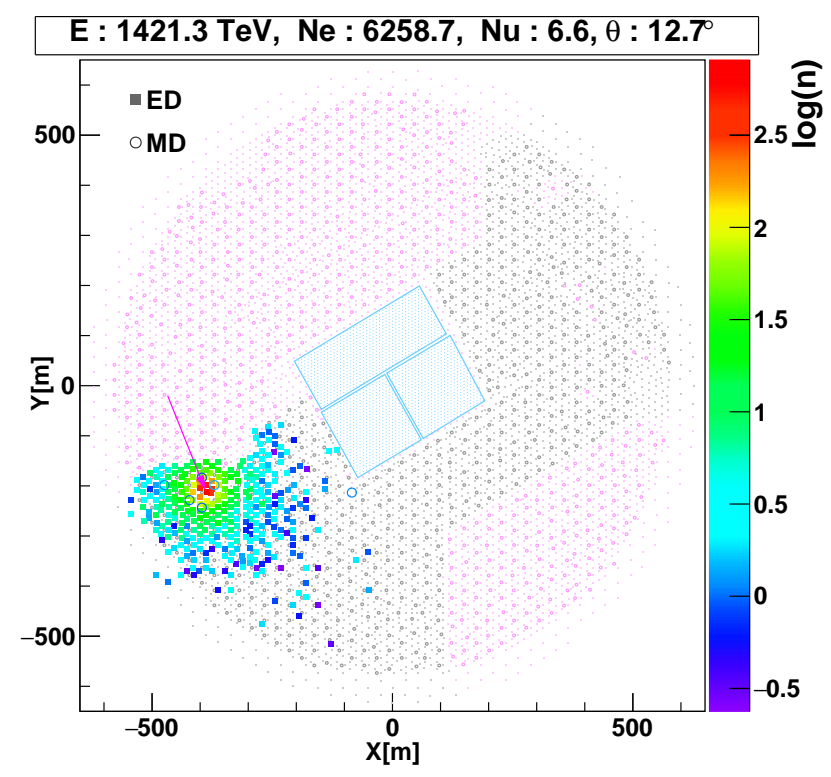

Figure 2: The charge distribution for the highest gamma-ray event from Cygnus Cocoon region

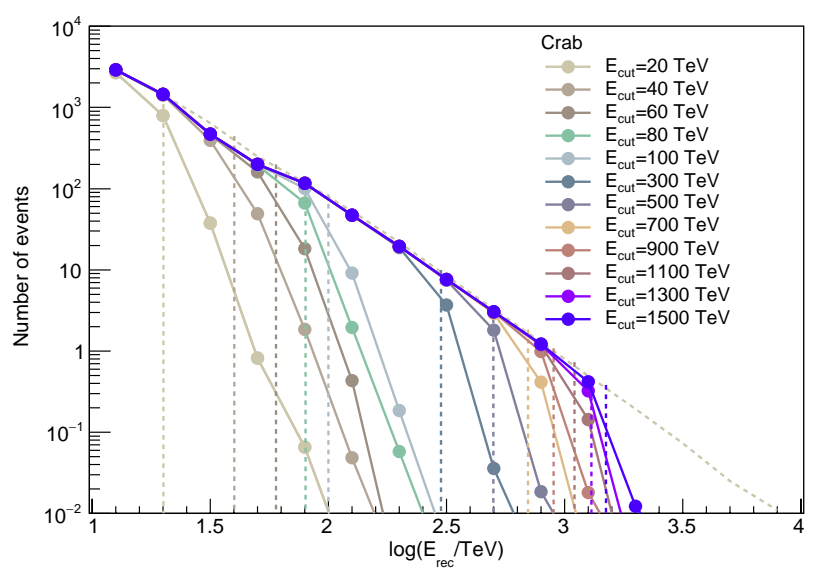

Figure 3: Distributions (dots) of events in the reconstructed energy bins from $10 \mathrm{TeV}$ to $10 \mathrm{PeV}$.

beyond PeV in superbubble. However, more data and detailed analysis are needed to give solid conclusion about this.

\section{References}

[1] Ackermann et al. 2013, Science, 339, 807

[2] Aharonian, F., Yang, R., \& de Oña Wilhelmi, E. 2019, Nature Astronomy, 3, 561

[3] Bykov, A. M. 2001, SSRv, 99, 317 
[4] Kalyashova, M. E., Bykov, A. M., Osipov, S. M., Ellison, D. C., \& Badmaev, D. V. 2019, in Journal of Physics Conference Series, Vol. 1400, Journal of Physics Conference Series, 022011

[5] Ackermann, M., Ajello, M., Allafort, A., et al. 2011, Science, 334, 1103

[6] Bartoli, B., Bernardini, P., Bi, X. J., et al. 2014, ApJ, 790, 152

[7] Abeysekara, A. U., Albert, A., Alfaro, R., et al. 2021, Nature Astronomy, doi:10.1038/s41550021-01361-9

[8] Aliu, E., Archambault, S., Arlen, T., et al. 2013, ApJ, 770, 93

[9] Aliu, E., Aune, T., Behera, B., et al. 2014, ApJ, 783, 16

[10] He, H. 2018, Radiation Detection Technology and Methods, 2

[11] Lv, H., He, H., Sheng, X., et al. 2018, Astroparticle Physics, 100, 22

[12] Aharonian, F., An, Q., Axikegu, et al. 2021, Chinese Physics C, 45, 025002

[13] Wilks, S. S. 1938, Ann. Math. Statist., 9, 60

[14] Fleysher, R., Fleysher, L., Nemethy, P., Mincer, A. I., \& Haines,T. J. 2004, ApJ, 603, 355

[15] Aharonian, F., An, Q., Axikegu, et al. 2020, arXiv e-prints, arXiv:2010.06205.

[16] Amenomori, M., Bao, Y. W., Bi, X. J., et al. 2019, PRL, 123, 051101

[17] Kelner, S. R., Aharonian, F. A., \& Bugayov, V. V. 2006, PRD, 74, 034018

[18] Cao, Z., Aharonian, F. A., An, Q., et al. 2021, Nature, 594, 33 


\section{Full Authors List: LHAASO Collaboration}

Zhen $\mathrm{Cao}^{1,2,3}$, F. Aharonian ${ }^{4,5}$, Q. An ${ }^{6,7}$, Axikegu ${ }^{8}$, L.X. Bai ${ }^{9}$, Y.X. Bai ${ }^{1,3}$, L.X. Bai ${ }^{9}$, Y.X. Bai ${ }^{1,3}$, Y.W. Bao ${ }^{10}$, D. Bastieri ${ }^{11}$, X.J. Bi ${ }^{1,2,3}$, Y.J. Bi ${ }^{1,3}$, H. Cai ${ }^{12}$, J.T. Cai ${ }^{11}$, Zhe Cao ${ }^{6,7}$, J. Chang ${ }^{13}$, J.F. Chang ${ }^{1,3,6}$, B.M. Chen ${ }^{14}$, E.S. Chen ${ }^{1,2,3}$, J. Chen ${ }^{9}$, Liang Chen $^{1,2,3}$, Liang Chen ${ }^{15}$, Long $\mathrm{Chen}^{8}$, M.J. Chen ${ }^{1,3}$, M.L. Chen ${ }^{1,3,6}$, Q.H. Chen $^{8}$, S.H. Chen ${ }^{1,2,3}$, S.Z. Chen ${ }^{1,3}$, T.L. Chen ${ }^{16}$,X.L. Chen $^{1,2,3}$, Y. Chen ${ }^{10}$, N. Cheng ${ }^{1,3}$, Y.D. Cheng ${ }^{1,3}$, S.W. Cui ${ }^{14}$, X.H. Cuii ${ }^{17}$, Y.D. Cui ${ }^{18}$, B. D'Ettorre Piazzoli1 ${ }^{19}$, B.Z. Dai ${ }^{20}$, H.L. Dai $^{1,3,6}$, Z.G. Dai ${ }^{7}$, Danzengluobu ${ }^{16}$, D. della Volpe ${ }^{21}$, X.J. Dong ${ }^{1,3}$, K.K. Duan ${ }^{13}$, J.H. Fan $^{11}$, Y.Z. Fan ${ }^{13}$, Z.X. Fan ${ }^{1,3}$, J. Fang ${ }^{20}$, K. Fang $^{1,3}$, C.F. Feng ${ }^{22}$, L. Feng ${ }^{13}$, S.H. Feng ${ }^{1,3}$, Y.L. Feng ${ }^{13}$, B. Gao ${ }^{1,3}$, C.D. Gao ${ }^{22}$, L.Q. Gao ${ }^{1,2,3}$, Q. Gao ${ }^{16}$, W. Gao ${ }^{22}$, M.M. Ge ${ }^{20}$, L.S. Geng ${ }^{1,3}$, G.H. Gong ${ }^{23}$, Q.B. Gou ${ }^{1,3}$, M.H. Gu ${ }^{1,3,6}$, F.L. Guo ${ }^{15}$, J.G. Guo ${ }^{1,2,3}$, X.L. Guo ${ }^{8}$, Y.Q. Guo ${ }^{1,3}$, Y.Y. Guo ${ }^{1,2,3,13}$, Y.A. $\mathrm{Han}^{24}$, H.H. He ${ }^{1,2,3}$, H.N. He ${ }^{13}$, J.C. He ${ }^{1,2,3}$, S.L. He ${ }^{11}$, X.B. He ${ }^{18}$, Y. He ${ }^{8}$, M. Heller ${ }^{21}$, Y.K. Hor ${ }^{18}$, C. Hou ${ }^{1,3}$, H.B. Hu ${ }^{1,2,3}$, S.

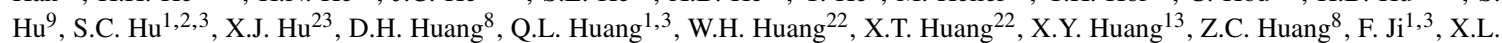
$\mathrm{Ji}^{1,3,6}$, H.Y. Jia ${ }^{8}$, K. Jiang ${ }^{6,7}$, Z.J. Jiang ${ }^{20}$, C. Jin ${ }^{1,2,3}$, T. Ke ${ }^{1,3}$, D. Kuleshov ${ }^{25}$, K. Levochkin ${ }^{25}$, B.B. Li ${ }^{14}$, Cheng Li ${ }^{6,7}$, Cong Li ${ }^{1,3}$,

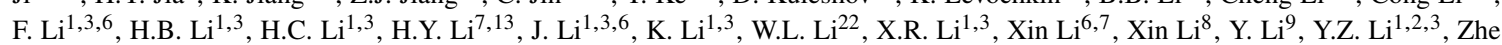

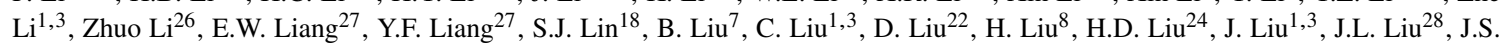

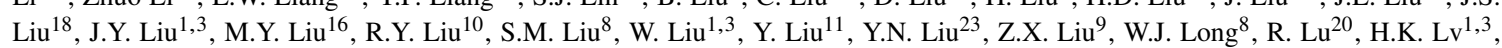

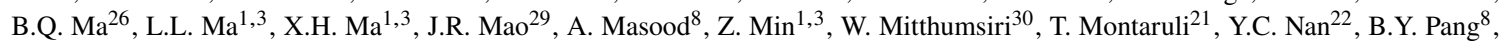
P. Pattarakijwanich ${ }^{30}$, Z.Y. Pei ${ }^{11}$, M.Y. Qi ${ }^{1,3}$, Y.Q. Qi ${ }^{14}$, B.Q. Qiao ${ }^{1,3}$, J.J. Qin ${ }^{7}$, D. Ruffolo ${ }^{30}$, V. Rulev ${ }^{25}$, A. Sáiz ${ }^{30}$, L. Shao ${ }^{14}$, O. Shchegolev $^{25,31}$, X.D. Sheng ${ }^{1,3}$, J.Y. Shi ${ }^{1,3}$, H.C. Song ${ }^{26}$, Yu.V. Stenkin ${ }^{25,31}$, V. Stepanov ${ }^{25}$, Y. Su ${ }^{32}$, Q.N. Sun ${ }^{8}$, X.N. Sun ${ }^{27}$, Z.B.

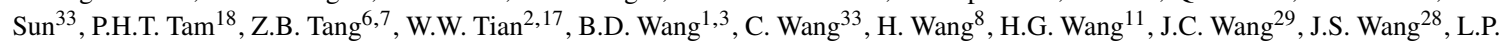
Wang $^{22}$, L.Y. Wang ${ }^{1,3}$, R.N. Wang ${ }^{8}$, W. Wang ${ }^{18}$, W. Wang ${ }^{12}$, X.G. Wang ${ }^{27}$, X.J. Wang ${ }^{1,3}$, X.Y. Wang ${ }^{10}$, Y. Wang ${ }^{8}$, Y.D. Wang ${ }^{1,3}$, Y.J. Wang $^{1,3}$, Y.P. Wang ${ }^{1,2,3}$, Z.H. Wang ${ }^{9}$, Z.X. Wang ${ }^{20}$, Zhen Wang ${ }^{28}$, Zheng Wang ${ }^{1,3,6}$, D.M. Wei ${ }^{13}$, J.J. Wei ${ }^{13}$, Y.J. Wei ${ }^{1,2,3}$, T. Wen $^{20}$, C.Y. $\mathrm{Wu}^{1,3}$, H.R. $\mathrm{Wu}^{1,3}$, S. $\mathrm{Wu}^{1,3}$, W.X. $\mathrm{Wu}^{8}$, X.F. Wu ${ }^{13}$, S.Q. Xi ${ }^{1,3}$, J. Xia ${ }^{7,13}$, J.J. Xia ${ }^{8}$, G.M. Xiang ${ }^{2,15}$, D.X. Xiao ${ }^{16}$, G. Xiao ${ }^{1,3}$, H.B. Xiao ${ }^{11}$, G.G. Xin ${ }^{12}$, Y.L. Xin ${ }^{8}$, Y. Xing ${ }^{15}$, D.L. Xu ${ }^{28}$, R.X. Xu ${ }^{26}$, L. Xue ${ }^{22}$, D.H. Yann ${ }^{29}$, J.Z. Yan ${ }^{13}$, C.W. Yang 9 , F.F. Yang ${ }^{1,3,6}$, J.Y. Yang ${ }^{18}$, L.L. Yang ${ }^{18}$, M.J. Yang ${ }^{1,3}$, R.Z. Yang ${ }^{7}$, S.B. Yang ${ }^{20}$, Y.H. Yao ${ }^{9}$, Z.G. Yao ${ }^{1,3}$, Y.M. Ye ${ }^{23}$, L.Q. Yin ${ }^{1,3}$, N. Yinn ${ }^{22}$, X.H. You $^{1,3}$, Z.Y. You ${ }^{1,2,3}$, Y.H. Yu ${ }^{22}$, Q. Yuan ${ }^{13}$, H.D. Zeng ${ }^{13}$, T.X. Zeng ${ }^{1,3,6}$, W. Zeng ${ }^{20}$, Z.K. Zeng ${ }^{1,2,3}$, M. Zha ${ }^{1,3}$, X.X. Zhai ${ }^{1,3}$, B.B. Zhang $^{10}$, H.M. Zhang ${ }^{10}$, H.Y. Zhang ${ }^{22}$, J.L. Zhang ${ }^{1}$, J.W. Zhang ${ }^{9}$, L.X. Zhang ${ }^{11}$, Li Zhang ${ }^{20}$, Lu Zhang ${ }^{14}$, P.F. Zhang ${ }^{20}$, P.P. Zhang ${ }^{14}$, R. Zhang ${ }^{7,13}$, S.R. Zhang ${ }^{14}$, S.S. Zhang ${ }^{1,3}$, X. Zhang ${ }^{10}$, X.P. Zhang ${ }^{1,3}$, Y.F. Zhang ${ }^{8}$, Y.L. Zhang ${ }^{1,3}$, Yi Zhang ${ }^{1,13}$, Yong Zhang ${ }^{1,3}$, B. Zhao $^{8}$, J. Zhao ${ }^{1,3}$, L. Zhao ${ }^{6,7}$, L.Z. Zhao ${ }^{14}$, S.P. Zhao ${ }^{13,22}$, F. Zheng ${ }^{33}$, Y. Zheng ${ }^{8}$, B. Zhou ${ }^{1,3}$, H. Zhou ${ }^{28}$, J.N. Zhou ${ }^{15}$, P. Zhou ${ }^{10}$, R. Zhou $^{9}$, X.X. Zhou ${ }^{8}$, C.G. Zhu ${ }^{22}$, F.R. Zhu ${ }^{8}$, H. Zhu ${ }^{17}$, K.J. Zhu ${ }^{1,2,3,6}$ and X. Zuo ${ }^{1,3}$

${ }^{1}$ Key Laboratory of Particle Astrophyics \& Experimental Physics Division \& Computing Center, Institute of High Energy Physics, Chinese Academy of Sciences, 100049 Beijing, China. ${ }^{2}$ University of Chinese Academy of Sciences, 100049 Beijing, China. ${ }^{3}$ TIANFU Cosmic Ray Research Center, Chengdu, Sichuan, China. ${ }^{4}$ Dublin Institute for Advanced Studies, 31 Fitzwilliam Place, 2 Dublin, Ireland. ${ }^{5}$ Max-Planck-Institut for Nuclear Physics, P.O. Box 103980, 69029 Heidelberg, Germany. ${ }^{6}$ State Key Laboratory of Particle Detection and Electronics, China. ${ }^{7}$ University of Science and Technology of China, 230026 Hefei, Anhui, China. ${ }^{8}$ School of Physical Science and Technology \& School of Information Science and Technology, Southwest Jiaotong University, 610031 Chengdu, Sichuan, China. ${ }^{9}$ College of Physics, Sichuan University, 610065 Chengdu, Sichuan, China. ${ }^{10}$ School of Astronomy and Space Science, Nanjing University, 210023 Nanjing, Jiangsu, China. ${ }^{11}$ Center for Astrophysics, Guangzhou University, 510006 Guangzhou, Guangdong, China. ${ }^{12}$ School of Physics and Technology, Wuhan University, 430072 Wuhan, Hubei, China. ${ }^{13}$ Key Laboratory of Dark Matter and Space Astronomy, Purple Mountain Observatory, Chinese Academy of Sciences, 210023 Nanjing, Jiangsu, China. ${ }^{14}$ Hebei Normal University, 050024 Shijiazhuang, Hebei, China. ${ }^{15}$ Key Laboratory for Research in Galaxies and Cosmology, Shanghai Astronomical Observatory, Chinese Academy of Sciences, 200030 Shanghai, China. ${ }^{16}$ Key Laboratory of Cosmic Rays (Tibet University), Ministry of Education, 850000 Lhasa, Tibet, China. ${ }^{17}$ National Astronomical Observatories, Chinese Academy of Sciences, 100101 Beijing, China. ${ }^{18}$ School of Physics and Astronomy \& School of Physics (Guangzhou), Sun Yat-sen University, 519000 Zhuhai, Guangdong, China. ${ }^{19}$ Dipartimento di Fisica dell'Università di Napoli ‘Federico II”, Complesso Universitario di Monte Sant'Angelo, via Cinthia, 80126 Napoli, Italy. ${ }^{20}$ School of Physics and Astronomy, Yunnan University, 650091 Kunming, Yunnan, China. ${ }^{21}$ D'epartement de Physique Nucl'eaire et Corpusculaire, Facult'e de Sciences, Universit'e de Gen'eve, 24 Quai Ernest Ansermet, 1211 Geneva, Switzerland. ${ }^{22}$ Institute of Frontier and Interdisciplinary Science, Shandong University, 266237 Qingdao, Shandong, China. ${ }^{23}$ Department of Engineering Physics, Tsinghua University, 100084 Beijing, China. ${ }^{24}$ School of Physics and Microelectronics, Zhengzhou University, 450001 Zhengzhou, Henan, China. ${ }^{25}$ Institute for Nuclear Research of Russian Academy of Sciences, 117312 Moscow, Russia. ${ }^{26}$ School of Physics, Peking University, 100871 Beijing, China. ${ }^{27}$ School of Physical Science and Technology, Guangxi University, 530004 Nanning, Guangxi, China. ${ }^{28}$ Tsung-Dao Lee Institute \& School of Physics and Astronomy, Shanghai Jiao Tong University, 200240 Shanghai, China. ${ }^{29}$ Yunnan Observatories, Chinese Academy of Sciences, 650216 Kunming, Yunnan, China. ${ }^{30}$ Department of Physics, Faculty of Science, Mahidol University, 10400 Bangkok, Thailand. ${ }^{31}$ Moscow Institute of Physics and Technology, 141700 Moscow, Russia. ${ }^{32}$ Key Laboratory of Radio Astronomy, Purple Mountain Observatory, Chinese Academy of Sciences, 210023 Nanjing, Jiangsu, China. ${ }^{33}$ National Space Science Center, Chinese Academy of Sciences, 100190 Beijing, China. 